\title{
Ultrafast Nonlinear Fiber Optics: Single-Pulse Supercontinua and Specialty-Shape Pulses
}

\author{
Igor A. Sukhoivanov, ${ }^{1}$ Gabriel Ramos Ortiz, ${ }^{2}$ Oscar Ibarra Manzano ${ }^{1}$, \\ J. Amparo Andrade Lucio ${ }^{1}$, Oleksiy V. Shulika ${ }^{* 1}$ \\ ${ }^{1}$ División de Ingenierías, Universidad de Guanajuato, Carretera Salamanca - Valle de Santiago Km. 3.5 + 1.8., \\ 36885 Salamanca, México \\ ${ }^{2}$ Centro de Investigaciónes en Óptica, Loma del Bosque 115, León 37150, México
}

Received February 20, 2015; accepted March 31, 2015; published March 31, 2015

\begin{abstract}
Needs of science and technology for compact laser sources with specially shaped pulse waveforms or an ultra-broad spectrum can be satisfied on the fiber-optic platform. Here we address the transformation of ultrashort optical pulses in a variety of optical fibers aiming for a synthesis of specially shaped pulses and single-pulse flat-top supercontinuum. The results are discussed in the context of possible applications and experimental implementations.
\end{abstract}

Ultra broad spreading of ultrashort pulses propagating in an optical fibre involves a variety of nonlinear phenomena. Great interest in this field was inspired by the invention of microstructured optical fibres (MOFs), which have allowed supercontinum (SC) generation for a much wider range of source parameters than was possible with bulk media or conventional fibres. Despite the great potential of MOFs for controlling the dispersive and nonlinear properties of the guiding medium, most research has concentrated on pumping in the anomalous dispersion region [1]. These studies have uncovered key contributions of soliton fission and modulation instability to SC generation under pumping in the anomalous dispersion regime. In turn, these results have allowed the invention of other methods of SC generation such as CW pumping and pumping with nano-/pico-second pulses [1].

The broadening mechanism of the SC generated in anomalous dispersion is dominated by soliton dynamics and soliton fission, which are sensitive to input pulse fluctuations and pump laser shot noise. Another drawback of this type of SC is the lack of coherence and the fact that in the time domain, it does not exhibit a single-pulse waveform but has the form of a noise-like train of spikes. An alternative to the pumping in anomalous dispersion region is pumping in the normal dispersion region, which could benefit from the generation of octave-spanning supercontinua, which are characterized by the preservation of a single-pulse waveform in the time domain, perfect temporal coherence and spectral properties [2-4] suitable for efficient external recompression. This is important because of the practical

*E-mail: oshulika@ugto.mx demands of few-cycle laser pulses in time-resolved studies of fundamental processes in physics, chemistry, and biology. Although these applications drive the development of various techniques for generating fewcycle pulses, the generation of high quality few-cycle pulses by inexpensive and simple methods is still challenging.

The transformation of pulse shapes is another type of nonlinear phenomenon that occurs in the normal dispersion regime. It provides a simple option for the transformation of pulse waveforms needed for many practical applications. Flat-top (rectangular-like), triangular, and parabolic pulses are used in all-optical signal processing, ultra-high-speed optical systems, and nonlinear optics [5].

In particular, the interest in parabolic pulses in recent

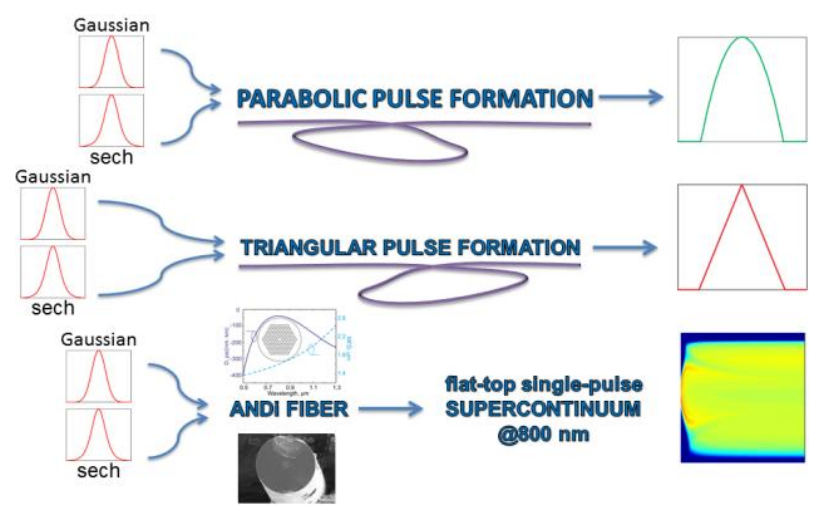

Fig.1. Three situations presented in manuscript.

years was inspired by their unique properties [6]. These pulses propagate self-similarly in an active fibre in the presence of normal dispersion and nonlinearity; therefore, they are called similaritons. The amplitude and width scaling in this case depend only on amplifier parameters and input pulse energy. Parabolic pulses have found numerous applications in pulse amplification and compression [7] as well as in optical communications [5, 7]. These include optical regeneration [8], pulse re-timing 

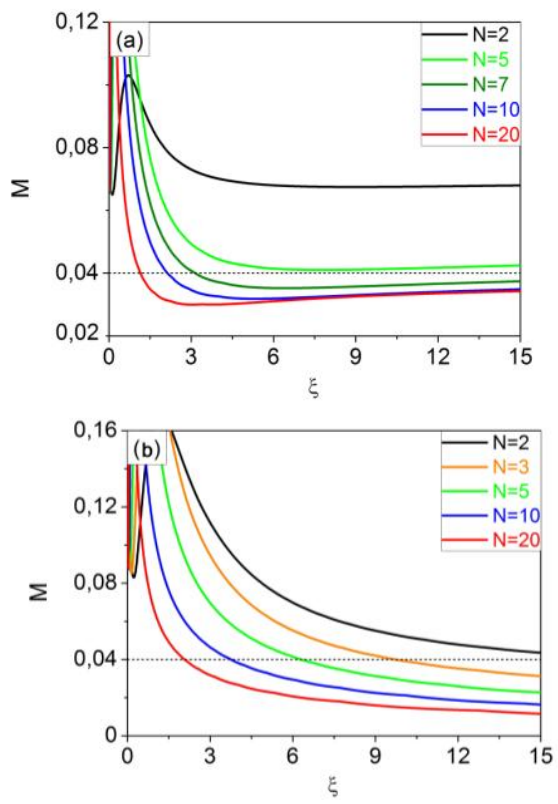

Fig. 2. Evolution of the misfit parameter $M$ versus normalized length $\xi$ under incidence of Gaussian (a) secant (b) pulses. The case of triangular pulse formation.

[9], optimization of return-to-zero optical receivers [10], and the mitigation of linear waveform distortions [11]. It was shown, however, that parabolic pulses as well as triangular ones, can be formed in a passive optical fiber [12-14].

Another very interesting case of passive nonlinear
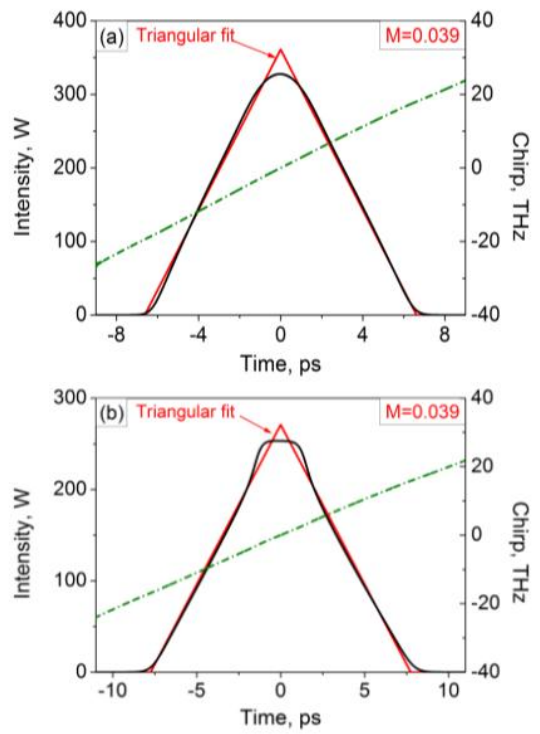

Fig. 3. Triangular pulses produced in the Thorlabs $780 \mathrm{HP}$ fiber. a) temporal intensity and chirp (green curve) for triangular pulse generated at the fiber length $1.4527 \mathrm{~m}$ from initial Gaussian pulse $\left(N=7, E_{0}=2.39\right.$ nJ, FWHM=200fs $)$; b) temporal intensity and chirp (green curve) for triangular pulse generated at the fiber length $1.9435 \mathrm{~m}$ from initial secant pulse $\left(N=6, E_{0}=2.101 \mathrm{~nJ}\right.$, FWHM=200fs) reshaping, which we report here, is generation of singlepulse SC. The term "single-pulse" refers to the unique feature: conservation of the pumping pulse as such, during its nonlinear ultrabroad spreading in the Fourier domain. As compared to the traditional SC generation, in the last case a highly coherent, flat-top spectrum can be generated preserving a single pulse waveform in the temporal domain, which is rather beneficial for applications. All three cases reported here are shown in Fig. 1.

The evolution of an ultrashort pulse during its propagation in a normal-dispersion optical fiber with Kerr nonlinearity is studied by solving nonlinear Schrödinger equation. Figure 2 shows the evolution of the misfit parameter $M$ versus normalized length $\xi$ for initial unchirped Gaussian and secant pulse shapes for different values of soliton order N. Misfit parameter M shows the deviation from ideal triangular profile; it is considered that pulse is close enough to the triangular waveform when $\mathrm{M}<0.04$.

Figure 2 shows that a triangular pulse shape can be achieved both from Gaussian and secant initial pulses. The condition for Gaussian pulse is $\mathrm{N}>7$, whereas for secant pulse $\mathrm{N}>3$. These conditions provide the formation of triangular pulses which preserve a triangular shape during the propagation in the fiber as Fig. 2 shows. Using these results we have calculated the actual pulse shapes obtained from initial Gaussian and secant pulses after propagation in conventional single mode fiber Thorlabs $780 \mathrm{HP}$, which has normal dispersion at $800 \mathrm{~nm}$.
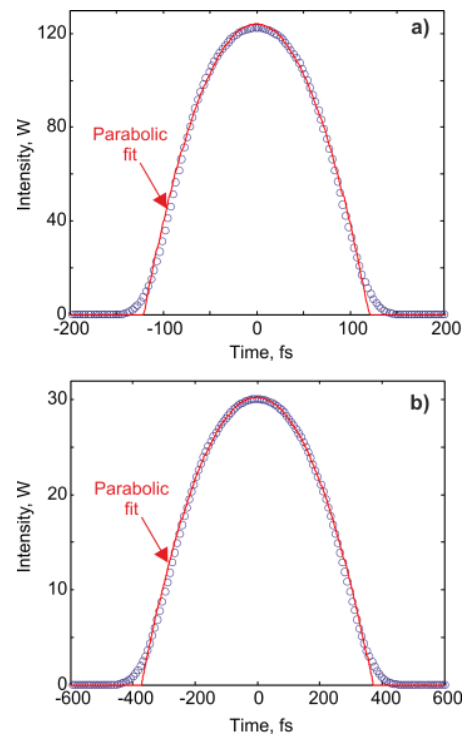

Fig. 4. Parabolic pulses generated in the ANDi PCF (circles) and appropriate parabolic fitting curves. a) - parabolic pulse generated from initial unchirped Gaussian pulse in the transient regime ( $\left.\tau_{0}=80 \mathrm{fs}, E_{0}=20 \mathrm{pJ}, z=10 \mathrm{~cm}\right)$, misfit parameter is $\left.M=0.035 . \mathrm{b}\right)-$ parabolic pulse generated from initial chirped Gaussian pulse in the steady-state regime ( $\left.\tau_{0}=80 \mathrm{fs}, E_{0}=15 \mathrm{pJ}, C=1.24, z=30 \mathrm{~cm}\right)$, misfit parameter is $M=0.033$. 

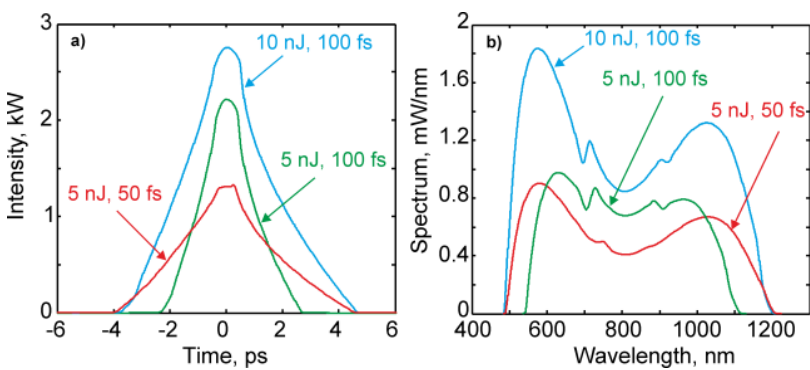

Fig. 5. Supercontinuum generation in ANDi-MOF of $10 \mathrm{~cm}$ length for different pulse energies and durations. a) - pulse profiles in temporal domain; b) - corresponding pulse spectra.

From Fig. 3 one can see that pulse shape indeed is close to the triangular waveform, moreover the chirp of the pulses is perfectly linear.

Figure 4 shows examples of parabolic pulses generated via passive nonlinear reshaping in all-normal dispersion microstructured optical fiber (ANDi-MOF) in the transient regime and in the steady-state regime. We can see that both pulses are perfectly parabolic. However, at the short propagation distance $(10 \mathrm{~cm})$ pulse width (FWHM) is $167 \mathrm{fs}$; in the steady-state regime $(30 \mathrm{~cm})$ it is larger, 513fs, due to the longer propagation distance in the fiber required for appearance of the steady-state regime.

Figure 5 shows the simulation results of SC generation in the designed ANDi-MOF [opex] using $10 \mathrm{~cm}$ fiber piece and initial Gaussian pulses with different pulse energy and duration. Figure 5a) shows that during SC generation a single pulse shape remains in this fiber. Figure 5b) shows that the best flatness of the SC spectrum is achieved for smaller pulse energy $-5 \mathrm{~nJ}$ and larger pulse duration - 100 fs. However, the spectral width in this case is smaller $\sim 572 \mathrm{~nm}$. If we increase initial pulse energy up to $10 \mathrm{~nJ}$, spectral width is increased up to $710 \mathrm{~nm}$ corresponding to $\sim 1.3$ octave. Spectral width is also increased up to $711 \mathrm{~nm}$, if we use initial pulses with smaller duration - 50fs.

Thus, increasing initial pulse energy or application of shorter initial pulses allows obtaining wider spectral width. However, the price for that is worse spectral flatness. Additional spectral broadening is accompanied by the depletion of the central part of the spectrum, such

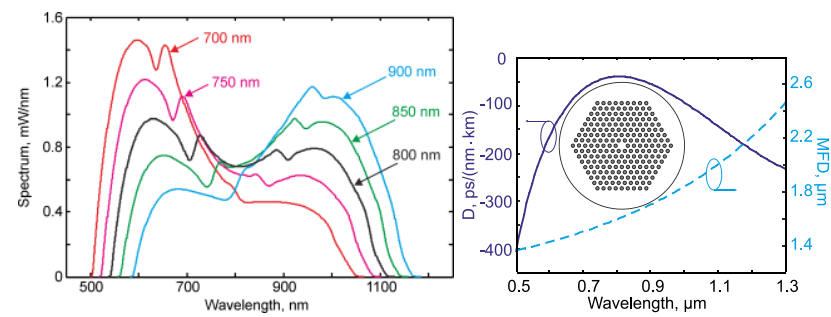

Fig. 6. Supercontinuum spectra generated in ANDi-MOF of $10 \mathrm{~cm}$ length for different pump wave-lengths. In all cases initial pulse energy is $5 \mathrm{~nJ}$, pulse duration is $100 \mathrm{fs}$. Dispersion curves of the fiber are aslo shown. that a dip appears at the pumping wavelength

Spectral changes appearing during the shifting of the incident pulse wavelength are shown in Fig. 6, showing that the wavelength range which is optimal for pumping does exist and is located around the extremum of the fiber dispersion spectrum, which is $\sim 760 \mathrm{~nm}<\lambda_{\mathrm{p}}<840 \mathrm{~nm}$ for our fiber.

To conclude, we have discussed nonlinear pulse reshaping towards parabolic and triangular waveforms, and supercontinuum generation in normally dispersive optical fibers. Conditions for triangular pulse formation in the steady-state regime have been presented. The conditions of maximal spectral broadening while keeping good spectral flatness in specially designed ANDi-MOF have been presented as well.

This work is partially supported by University of Guanajuato (projects DAIP-633/2015 and DAIP609/2015).

\section{References}

[1] J.M. Dudley, G. Genty, S. Coen, Rev. Mod. Phys. 78(4), 1135 (2006). http://dx.doi.org/10.1103/RevModPhys.78.1135

[2] A.M. Heidt, A. Hartung, G. W. Bosman, P. Krok, E.G. Rohwer, H. Schwoerer, H. Bartelt, Opt. Expr. 19(4), 3775 (2011). http://dx.doi.org/10.1364/OE.19.003775

[3] A.M. Heidt, J. Rothhardt, A. Hartung, H. Bartelt, E.G. Rohwer, J. Limpert, A. Tünnermann, Opt. Expr. 19, 13873 (2011). http://dx.doi.org/10.1364/OE.19.013873

[4] A. Hartung, A.M. Heidt, H. Bartelt, Opt. Expr. 19, 7742 (2011) http://dx.doi.org/10.1364/OE.19.007742

[5] S. Boscolo, C. Finot, Intern. J. Optics 2012, 159057 (2012). http://dx.doi.org/10.1155/2012/159057

[6] J.M. Dudley, C. Finot, D.J. Richardson, G. Millot, Nature Phys. 3, 597 (2007). http://dx.doi.org/10.1038/nphys705

[7] C. Finot, J.M. Dudley, B. Kibler, D.J. Richardson, G. Millot, IEEE J. Quantum Electron. 45, 1482 (2009). http://dx.doi.org/10.1109/JQE.2009.2027446

[8] C. Finot, S. Pitois, G. Millot, Opt. Lett. 30, 1776 (2005) http://dx.doi.org/10.1364/OL.30.001776

[9] F. Parmigiani, P. Petropoulos, M. Ibsen, D.J. Richardson, IEEE Photon. Technol. Lett. 18, 829 (2006). http://dx.doi.org/10.1109/LPT.2006.871848

[10] S. Boscolo, S.K. Turitsyn, K.J. Blow, Opt. Expr. 13, 6217 (2005) http://dx.doi.org/10.1364/OPEX.13.006217

[11] T.T. Ng, F. Parmigiani, M. Ibsen, Z. Zhang, P. Petropoulos, D.J. Richardson, IEEE Photon. Technol. Lett. 20, 1097 (2008). http://dx.doi.org/10.1109/LPT.2008.924304

[12] S.O. Iakushev, O.V. Shulika, I.A. Sukhoivanov, Opt. Commun. 285, 4493 (2012). http://dx.doi.org/10.1016/j.optcom.2012.06.024

[13] I.A. Sukhoivanov, S.O. Iakushev, O.V. Sulika, A. Díez, M. Andrés, Opt. Expr., 21, 17769 (2013). http://dx.doi.org/10.1364/OE.21.017769

[14] S.O. Iakushev, O.V. Shulika, I.A. Sukhoivanov, V.I. Fesenko, M.V. Andres, H. Sayinc, Opt. Expr. 22(23), 29119 (2014). http://dx.doi.org/10.1364/OE.22.029119 